\title{
Ingestão de açúcar de adição por estudantes da área da saúde em uma instituição de ensino de Viçosa, Brasil
}

\author{
Added sugar intake of health area students in an educational
} institution of Viçosa, Brazil

Dalila Pinto de Souza Fernandes* Flávia Galvão Cândido** José Luiz Marques Rocha*** Vanessa Guimarães Reis*** Eliana Carla Gomes de Souza*** Maria Sônia Lopes Duarte ${ }^{* * *}$

\begin{abstract}
Resumo
Evidências mostram que o consumo excessivo de açúcares, pode comprometer a qualidade da alimentação impactando negativamente na saúde. Assim, o objetivo do presente estudo foi avaliar o consumo de macronutrientes e adequação do consumo de açúcar por estudantes da área da saúde da Universidade Federal de Viçosa, Brasil. Participaram deste estudo 157 mulheres e 30 homens, com faixa etária de 21,3 \pm 3,4 anos, apresentando IMC e CC médios de 22,03 \pm 4,03 kg.m-2 e 72,95 \pm 8,96 cm, respectivamente. Foram aplicados questionários semiestruturados e procedeu-se às avaliações antropométricas e dietéticas dos indivíduos. Os resultados revelaram alta ingestão média diária de açúcar de adição $(69,21 \pm 5,25 \mathrm{~g})$ que, consequentemente, aumentou o teor de frutose da alimentação $(36,22 \pm 29,78 \mathrm{~g})$, além de uma baixa ingestão de fibras $(15,51 \pm 7,30 \mathrm{~g})$. Verificou-se que a ingestão média de açúcar de adição esteve significativamente $(p<0,05)$ associada ao peso corporal $(0,172 ; p=0,041)$, IMC $(0,181 ; p=0,031)$ e consumo dos macronutrientes e cálcio $(0,247 ; p=0,003)$. Deve-se considerar a necessidade de implementação de medidas que visem promover mudanças comportamentais importantes no que diz respeito à alimentação de graduandos dos cursos de saúde.
\end{abstract}

Palavras-chave: Açúcar. Estudantes. Consumo alimentar.

\begin{abstract}
Evidence shows that excessive sugar consumption may impair the quality of feeding affecting negatively on health. The objective of this study was evaluated the intake of macronutrients and adequacy of sugar consumption by healthcare students of Federal University of Viçosa, Brazil. The study included 157 women and 30 men, aged $21.3 \pm 3.4$ years, with mean body mass index (BMI) of $22.03 \pm 4.03 \mathrm{~kg} \mathrm{~m}-2$ and waist circumference (WC) of $72.95 \pm 8.96 \mathrm{~cm}$. Semistructured questionnaires were administered and proceeded to anthropometric and dietary assessments of individuals. The results revealed high average daily intake of added sugar ( $69.21 \pm 5.25 \mathrm{~g})$ which consequently increased the fructose content of feeding $(36.22 \pm 29.78 \mathrm{~g})$, and a low intake of fiber $(15.51 \pm 7.30 \mathrm{~g})$. It was found that the average intake of added sugar was significantly $(p<0.05)$ associated to body weight $(0.172, p=0.041), B M I(0.181, p=0.031)$ and consumption of macronutrients and calcium $(0.247 ; p=0.003)$. Should consider the need to implement measures to promote significant behavioral changes with regard to the supply of undergraduate health courses.
\end{abstract}

Keywords: Sugar. Students. Food consumption.

DOI: 10.15343/0104-7809.2016400194105

*Universidade Federal de Viçosa, Departamento de Nutrição e Saúde, Viçosa-MG, Brasil. E-mail: dalilaf.ufv@gmail.com **Universidade Federal de Viçosa, Departamento de Nutrição e Saúde, Viçosa-MG, Brasil. E-mail: flaviagcandido@hotmail.com

*** Universidade Federal de Viçosa - Departamento de Nutrição e Saúde, Viçosa-MG, Brasil.

**** Universidade Federal de Viçosa - Departamento de Nutrição e Saúde, Viçosa-MG, Brasil. E-mail: msonia.duarte@ufv.br Os autores declaram não haver conflitos de interesse. 


\section{INTRODUÇÃO}

O termo açúcar de adição refere-se a todo mono ou dissacarídeo acrescentado aos alimentos ou bebidas durante suas preparações ${ }^{1}$. Refrigerantes, sucos, biscoitos e doces são as principais fontes de açúcar simples da dieta ocidental ${ }^{2,3}$. Entre estes, os refrigerantes representaram a fonte mais significativa de açúcar simples das últimas três décadas, nos Estados Unidos ${ }^{3,4}$.

Atualmente, o consumo de açúcar de adição tem crescido a proporções elevadas em diversas populações ${ }^{1,2}$. Evidências científicas mostram que o consumo excessivo de açúcares, especialmente a sacarose, pode comprometer a qualidade da dieta impactando negativamente na saúde e na situação nutricional das populações de países desenvolvidos e em desenvolvimento ${ }^{5,6}$.

Diversos estudos demonstram a associação entre o consumo excessivo de açúcar simples e o desenvolvimento de doenças crônicas não transmissíveis como o Diabetes mellitus tipo 2 (DM2), a obesidade, as dislipidemias e as doenças cardiovasculares ${ }^{1,2,3}$. Acredita-se que o consumo desses alimentos, devido ao seu alto teor de frutose e elevadas cargas glicêmicas, aumente os riscos de doenças por conduzir ao ganho ponderal, aumentar a resistência insulínica e favorecer a síntese de ácidos graxos e os processos inflamatórios ${ }^{4,7,8}$.

Por todas as evidências, a Organização Mundial de Saúde (OMS) estabeleceu limites populacionais máximos para o consumo de açúcar em $10 \%$ do valor energético total (VET) (WHO, 2003), sendo este preconizado pelo Guia Alimentar para a População Brasileira, proposto pelo Ministério da Saúde ${ }^{9}$. O mesmo guia também orienta a redução do consumo de alimentos e bebidas processados, com alta concentração de açúcar e também a redução de sua adição nas preparações caseiras. Além disso, estabelece que o consumo máximo diário deva ser de uma porção de alimentos do grupo dos açúcares e doces. No entanto, de acordo com a última Pesquisa de Orçamentos Familiares, o valor estabelecido foi largamente ultrapassado em todas as regiões do Brasil (variando de 13,9\% das calorias totais na região norte a $17,4 \%$ na região sudeste) e em todas as classes sociais ${ }^{10}$.

O consumo alimentar de estudantes universitários é vulnerável 11,12 e fatores estressores relacionados ao novo ambiente acadêmico $^{13}$ são positivamente associados com a ingestão de alimentos densos, altamente calóricos com alto teor de açúcar e, alguns estudos 14,15,16 apontam que os universitários da área de saúde mantêm o mesmo comportamento alimentar de universitários de outras áreas.

Os estudantes da área da saúde são importantes alvos de pesquisa uma vez que se tornarão orientadores e conselheiros de pacientes nas estratégias de prevenção de doenças. Entretanto, poucos estudos têm priorizado a investigação das condutas de alimentação entre futuros profissionais de saúde para que os mesmos possam ser estimulados a adotarem práticas saudáveis.

Tendo em vista os impactos negativos na saúde humana, decorrentes do consumo excessivo de açúcar de adição, a inadequação desse consumo na população brasileira e a importância dos profissionais da saúde na disseminação de hábitos alimentares saudáveis, o presente artigo teve por objetivo avaliar o consumo de açúcares de adição e suas relações com consumo de nutrientes e variáveis socioeconômicas, demográficas e antropométricas em estudantes da área da saúde.

\section{METODOLOGIA}

\section{Sujeitos}

Foram convidados para participar do estudo 607 estudantes dos cursos de graduação de áreas da saúde da Universidade Federal de Viçosa (UFV) - Campus Viçosa/MG. Dos estudantes convidados ( $N=607 ; 100 \%){ }^{17}, 31 \%$ $(n=187)$ aceitaram participar da pesquisa.

O presente estudo foi aprovado pelo Comitê de Ética da Universidade Federal de Viçosa (Of. Ref. No 114/2011) e atende às determinações da Resolução 196/96 da Comissão Nacional
95 
de Ética e Pesquisa em Seres Humanos. Todos os voluntários foram devidamente esclarecidos e assinaram o termo de consentimento livre e esclarecido.

\section{Delineamento experimental}

Trata-se de um estudo do tipo transversal. Utilizou-se para a coleta de dados um questionário semiestruturado especificamente desenvolvido para a pesquisa. As variáveis avaliadas foram: idade, sexo, curso de graduação, período letivo e nível de atividade física. A categorização do nível de atividade física seguiu os critérios do Institute of Medicine ${ }^{18}$. Após a aplicação dos questionários, foram feitas as avaliações antropométrica e dietética dos indivíduos de maneira individualizada.

\section{Avaliação dietética}

Para a avaliação dietética, foi utilizado o método do recordatório habitual de 24 horas. O método consiste em obter minuciosamente as informações sobre as quantidades de alimentos consumidos habitualmente pelo indivíduo, em medidas caseiras. Para minimizar os erros de estimação de porções, as medidas caseiras foram baseadas no Registro Fotográfico para Inquéritos Dietéticos ${ }^{19}$. O recordatório foi realizado em três etapas de acordo com Jonhson et al. ${ }^{20}$.

Foram considerados açúcares de adição todos os açúcares adicionados aos alimentos durante o seu processamento ou preparo, além do açúcar adicionado ao alimento no momento do consumo. A análise do consumo de açúcar de cada indivíduo foi feita após a padronização da quantidade percentual de açúcar presente em cada alimento. Para isso, foi elaborada uma tabela contendo os principais alimentos fonte de açúcares de adição consumidos e seus respectivos teores de carboidrato total e de açúcares de adição, de onde se calculou o percentual de açúcar simples do alimento/ preparação.

Considerou-se o teor de frutose de adição como sendo a média de $52,5 \%$ do conteúdo de açúcar de adição baseados nos conteúdos de frutose da sacarose e do xarope de milho com alto teor de frutose ${ }^{21}$. As quantidades de carboidrato total, açúcar de adição e o percentual adotadas para a avaliação dietética no presente estudo encontram-se na Tabela 1.

Tabela 1. Teor de carboidratos totais e simples dos principais alimentos consumidos por estudantes de áreas da saúde de uma universidade pública, Viçosa/MG, 2013.

\begin{tabular}{|c|c|c|c|c|}
\hline Alimentos (100g) & CHO Total (g) & $\begin{array}{l}\text { Açúcar de } \\
\text { adição (g) }\end{array}$ & $\begin{array}{c}\text { \% Açúcar de } \\
\text { adição }\end{array}$ & $\begin{array}{r}\text { Frutose de } \\
\text { adição (g) }\end{array}$ \\
\hline \multicolumn{5}{|l|}{ Leite e Derivados } \\
\hline Leite fermentado ${ }^{1}$ & 15,70 & 15,70 & 100,00 & 8,24 \\
\hline $\begin{array}{l}\text { logurte } \\
\text { integral (natural) }{ }^{1}\end{array}$ & 9,10 & 9,10 & 100,00 & 4,78 \\
\hline $\begin{array}{l}\text { logurte desnatado } \\
\text { (natural) }{ }^{1}\end{array}$ & 9,20 & 9,20 & 100,00 & 4,83 \\
\hline $\begin{array}{l}\text { Achocolatado } \\
\text { de caixinha }{ }^{1}\end{array}$ & 16,00 & 14,50 & 90,63 & 7,61 \\
\hline $\begin{array}{l}\text { Vitamina } \\
\text { de caixinha }^{1}\end{array}$ & 14,21 & 14,21 & 100,00 & 7,46 \\
\hline Petit suisse $^{1}$ & 13,00 & 10,42 & 80,15 & 5,47 \\
\hline \multicolumn{5}{|l|}{ Cereais e derivados } \\
\hline Bolo simples ${ }^{2}$ & 56,74 & 30,00 & 52,87 & 15,75 \\
\hline
\end{tabular}

continua... 
...continuação - Tabela 1

\begin{tabular}{|c|c|c|c|c|}
\hline $\begin{array}{l}\text { Bolo de chocolate } \\
\text { (com cobertura) }^{2}\end{array}$ & 56,74 & 45,30 & 79,84 & 23,78 \\
\hline 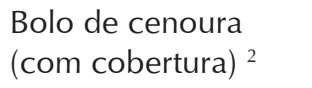 & 76,74 & 65,30 & 85,09 & 34,28 \\
\hline Bolo de aniversário $^{2}$ & 64,80 & 29,00 & 44,75 & 15,22 \\
\hline $\begin{array}{l}\text { Torta doce } \\
\text { (diversas) }^{2}\end{array}$ & 50,05 & 29,00 & 57,94 & 15,22 \\
\hline 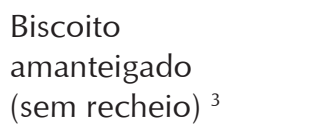 & 65,60 & 15,60 & 23,78 & 8,19 \\
\hline $\begin{array}{l}\text { Biscoito } \\
\text { tipo maisena }^{3}\end{array}$ & 75,00 & 25,00 & 33,33 & 13,12 \\
\hline $\begin{array}{l}\text { Biscoito recheado } \\
\text { (diversos) })^{1}\end{array}$ & 61,90 & 42,00 & 67,85 & 22,05 \\
\hline $\begin{array}{l}\text { Biscoito tipo wafer } \\
\text { (diversos) }{ }^{4}\end{array}$ & 71,60 & 43,60 & 60,89 & 22,89 \\
\hline Broa de milho ${ }^{2}$ & 46,60 & 21,00 & 45,06 & 11,02 \\
\hline Barrinha de cereal ${ }^{1}$ & 64,00 & 24,00 & 37,50 & 12,60 \\
\hline $\begin{array}{l}\text { Cereal matinal } \\
\text { (tipo Sucrilhos) }^{1}\end{array}$ & 86,67 & 40,00 & 46,15 & 21,00 \\
\hline Granola $^{1}$ & 65,00 & 42,50 & 65,38 & 22,31 \\
\hline $\begin{array}{l}\text { Rosquinha } \\
\text { (diversos) }^{3}\end{array}$ & 60,00 & 31,80 & 53,00 & 16,70 \\
\hline $\begin{array}{l}\text { Sequilhos } \\
\text { (diversos) }^{3}\end{array}$ & 85,71 & 25,71 & 30,00 & 13,50 \\
\hline \multicolumn{5}{|l|}{$\begin{array}{l}\text { Açúcares } \\
\text { e guloseimas }\end{array}$} \\
\hline Canjica doce $^{2}$ & 23,60 & 7,00 & 29,66 & 3,67 \\
\hline Arroz doce ${ }^{2}$ & 33,04 & 12,00 & 36,32 & 6,30 \\
\hline Sorvete ${ }^{1}$ & 26,70 & 26,70 & 100,00 & 14,02 \\
\hline Balas e doces ${ }^{3}$ & 99,50 & 99,50 & 100,00 & 52,24 \\
\hline $\begin{array}{l}\text { Pudim/manjar } \\
\text { de Leite }^{2}\end{array}$ & 38,00 & 21,80 & 57,37 & 11,44 \\
\hline $\begin{array}{l}\text { Chocolate } \\
\text { (diversos) }^{4}\end{array}$ & 59,60 & 56,00 & 93,96 & 29,40 \\
\hline Brigadeiro $^{2}$ & 112,00 & 112,00 & 100,00 & 58,80 \\
\hline $\begin{array}{l}\text { Sobremesas } \\
\text { tipo mousse }{ }^{2}\end{array}$ & 45,00 & 45,00 & 100,00 & 23,62 \\
\hline Rapadura $^{3}$ & 92,00 & 92,00 & 100,00 & 48,30 \\
\hline $\mathrm{Mel}^{3}$ & 84,00 & 84,00 & 100,00 & 44,10 \\
\hline 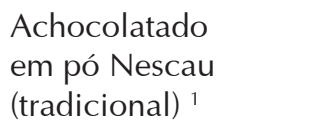 & 85,00 & 85,00 & 100,00 & 44,62 \\
\hline
\end{tabular}


...continuação - Tabela 1

\begin{tabular}{|c|c|c|c|c|}
\hline $\begin{array}{l}\text { Achocolatado em } \\
\text { pó Nescau (light) }{ }^{1}\end{array}$ & 71,58 & 71,58 & 100,00 & 37,58 \\
\hline $\begin{array}{l}\text { Achocolatado } \\
\text { em pó } \\
\text { Toddy (tradicional) }{ }^{1}\end{array}$ & 95,00 & 95,00 & 100,00 & 49,87 \\
\hline $\begin{array}{l}\text { Achocolatado } \\
\text { em pó Toddy } \\
\text { (light) }{ }^{1}\end{array}$ & 81,00 & 81,00 & 100,00 & 42,52 \\
\hline $\begin{array}{l}\text { Cappuccino em pó } \\
{\text { (tradicional })^{4}}\end{array}$ & 79,36 & 68,67 & 86,53 & 36,05 \\
\hline Doce de leite ${ }^{1}$ & 65,00 & 65,00 & 100,00 & 34,12 \\
\hline Pé de moleque ${ }^{2}$ & 58,80 & 58,80 & 100,00 & 30,87 \\
\hline Leite condensado $^{1}$ & 55,00 & 55,00 & 100,00 & 28,87 \\
\hline Paçoca $^{1}$ & 50,00 & 50,00 & 100,00 & 26,25 \\
\hline $\begin{array}{l}\text { Bombom } \\
\text { (diversos) }^{1}\end{array}$ & 65,00 & 65,00 & 100,00 & 34,12 \\
\hline $\begin{array}{l}\text { Goiabada } \\
\text { /mangada }^{2}\end{array}$ & 65,00 & 65,00 & 100,00 & 34,12 \\
\hline $\begin{array}{l}\text { Pêssego em calda/ } \\
\text { outros doces } \\
\text { em calda }^{2}\end{array}$ & 40,99 & 40,99 & 100,00 & 21,52 \\
\hline \multicolumn{5}{|l|}{ Bebidas } \\
\hline Café adoçado ${ }^{2}$ & 13,40 & 11,90 & 88,81 & 6,25 \\
\hline Chá adoçado ${ }^{2}$ & 13,40 & 13,40 & 100,00 & 7,03 \\
\hline $\begin{array}{l}\text { Café com } \\
\text { leite (tradicional) }{ }^{2}\end{array}$ & 8,64 & 5,00 & 57,87 & 2,62 \\
\hline $\begin{array}{l}\text { Refrigerante } \\
\text { tradicional } \\
\text { (diversos) }^{1}\end{array}$ & 10,76 & 10,76 & 100,00 & 5,65 \\
\hline $\begin{array}{l}\text { Refrigerante } \\
\text { light (diversos) }{ }^{1}\end{array}$ & 0,15 & 0,15 & 100,00 & 0,08 \\
\hline $\begin{array}{l}\text { Suco } \\
\text { artificial tradicional } \\
\text { (diversos) }{ }^{1}\end{array}$ & 14,30 & 14,30 & 100,00 & 7,51 \\
\hline $\begin{array}{l}\text { Suco artificial } \\
\text { light (diversos) }{ }^{1}\end{array}$ & 4,20 & 4,20 & 100,00 & 2,20 \\
\hline $\begin{array}{l}\text { Suco natural } \\
\text { de caixinha } \\
\text { (diversos) }{ }^{1}\end{array}$ & 14,00 & 14,00 & 100,00 & 7,35 \\
\hline $\begin{array}{l}\text { Suco natural } \\
\text { de caixinha } \\
\text { light (diversos) }{ }^{1}\end{array}$ & 3,50 & 3,50 & 100,00 & 1,84 \\
\hline $\begin{array}{l}\text { Suco de soja } \\
\text { de caixinha } \\
\text { (diversos) }^{1}\end{array}$ & 7,50 & 6,00 & 80,00 & 3,15 \\
\hline $\begin{array}{l}\text { Suco natural } \\
\text { adoçado } \\
\text { (diversos) }^{2}\end{array}$ & 17,41 & 17,41 & 100,00 & 9,14 \\
\hline
\end{tabular}

1 Tabela de Medidas Caseiras; 2 Rótulo dos Alimentos; 3 Receitas Internet; 4 Sites americanos. 
O cálculo da composição nutricional total dos alimentos consumidos foi realizada com o auxílio do Software DietPro (versão 5.5i). As preparações que envolveram mais de um tipo de alimento foram analisadas de acordo com as quantidades de cada ingrediente que a compôs. As análises foram conduzidas por avaliadores devidamente capacitados. O consumo de açúcares de adição foi categorizado, segundo contribuição percentual ao Valor Energético Total da Dieta (VET), em: adequado, quando $\leq 10 \% \mathrm{VET}$, e inadequado, quando $>10 \% \mathrm{VET}^{9}$.

A caracterização do padrão de consumo alimentar dos macronutrientes em relação ao VET foi realizada de acordo com o Guia Alimentar para a População Brasileira ${ }^{9}$, sendo 55\% a 75\% VET proveniente de carboidratos totais, 15\% a $30 \%$ de lipídios (gorduras saturadas devem ser inferiores a $10 \%$, gordura poli-insaturada de 6 a $10 \%$ e gordura monoinsaturada completa o percentual recomendado) e $10 \%$ a $15 \%$ de proteínas. Fibras alimentares, $25 \mathrm{~g} /$ dia e atividade física: $30 \mathrm{~min} / \mathrm{dia}$.

\section{Avaliação antropométrica}

O peso foi aferido em balança eletrônica (Tanita (C) BC554 IronMan) e o voluntário reduziu ao máximo o peso de acessórios. A medida da estatura realizada em estadiômetro Altura Exata, estando o voluntário descalço, de costas para o estadiômetro, em posição ereta, com os pés formando um ângulo de $45^{\circ}$. O Índice de Massa Corporal (IMC) e a classificação utilizada foram de acordo com os pontos de corte estabelecidos pela Organização Mundial de Saúde ${ }^{22}$.

Foram também aferidas a circunferência da cintura (CC) e circunferência do quadril (CQ), para o cálculo da relação cintura/quadril (RCQ) ${ }^{16}$. Segundo a WHO ${ }^{22}$ a RCQ indica risco de desenvolver doenças cardiovasculares, o ponto de corte estabelecido para homens é 1,0 e para mulheres é 0,85. A medida do perímetro da cintura foi aferida de acordo com os parâmetros preconizados pela $\mathrm{WHO}$, indicando risco aumentado para as DVC, de 90 $\mathrm{cm}$ para homens e $80 \mathrm{~cm}$ para as mulheres ${ }^{23}$.

\section{Análises estatísticas}

Após verificação da normalidade dos dados pelo teste Kolmogorov Smirnov, foi feita a análise de correlação de Pearson dos fatores associados à ingestão média de açúcar de adição. As análises foram conduzidas no software GraphPad Prism ${ }^{\circledR}$ V5.0. O nível de significância estatística adotado foi a<0,05.

\section{RESULTADOS E DISCUSSÃO}

Houve predomínio do gênero feminino $(83,83 \%) \quad(157$ mulheres e 30 homens), sendo que esses apresentaram faixa etária média de 21,3 $\pm 3,4$ anos. O predomínio do gênero feminino nos cursos da área de saúde é corroborado por vários estudos que se destinaram a estudar outros tipos de variáveis neste tipo de população ${ }^{24,25,26}$, uma vez que a área de saúde possui cursos culturalmente procurados mais por mulheres, como nutrição e enfermagem.

A avaliação antropométrica (Tabela 2), com baixos níveis de adiposidade central de acordo com as referências vigentes ${ }^{22,23}$. Os resultados encontrados são semelhantes ao estudo de Paixão et al. ${ }^{27}$, realizado com 253 estudantes ingressantes nos cursos de ciências da saúde em uma universidade do Recife, onde a média do IMC nos três cursos estudados também se encontra dentro da faixa de eutrofia. Vale ressaltar, a importância da adequação dos parâmetros antropométricos, uma vez que o excesso de gordura é prejudicial e relacionam com desordens cardiovasculares e metabólicas, causando grande impacto na saúde do indivíduo ${ }^{28}$.

A amostra possuiu distribuição pouco uniforme em relação ao número de indivíduos de cada curso e de cada período letivo. Fizeram parte da amostra 32 estudantes do curso de medicina $(17,11 \%$ do total), 79 do curso de nutrição (42,25\%) e 76 do curso de enfermagem (40,64\%). A distribuição dos estudantes em relação ao período letivo encontra-se descrita na Figura 1.

Diante do exposto, não foi viável a estratificação da amostra de acordo com o curso de graduação e o período letivo durante a análise dos dados. 
Tabela 2. Média, desvio padrão, erro padrão, mediana, valor mínimo e máximo das medidas antropométricas de estudantes da área de saúde, UFV, 2013.

\begin{tabular}{lcccccc}
\hline $\begin{array}{l}\text { Características } \\
(\mathbf{n}=\mathbf{1 8 7})\end{array}$ & Média & $\begin{array}{c}\text { Desvio } \\
\text { Padrão }\end{array}$ & $\begin{array}{c}\text { Erro } \\
\text { Padrão }\end{array}$ & Mediana & $\begin{array}{c}\text { Valor } \\
\text { Mínimo }\end{array}$ & $\begin{array}{c}\text { Valor } \\
\text { Máximo }\end{array}$ \\
\hline Idade (anos) & 21,30 & 3,42 & 0,251 & 21,00 & 17,00 & 45,00 \\
\hline Peso $(\mathrm{kg})$ & 60,90 & 12,20 & 0,89 & 58,80 & 41,10 & 112,50 \\
\hline IMC $(\mathrm{kg} \cdot \mathrm{m}-2)$ & 22,03 & 4,03 & 0,29 & 21,66 & 18,00 & 39,86 \\
\hline CC $(\mathrm{cm})$ & 72,95 & 8,96 & 0,66 & 70,40 & 58,60 & 106,00 \\
\hline
\end{tabular}

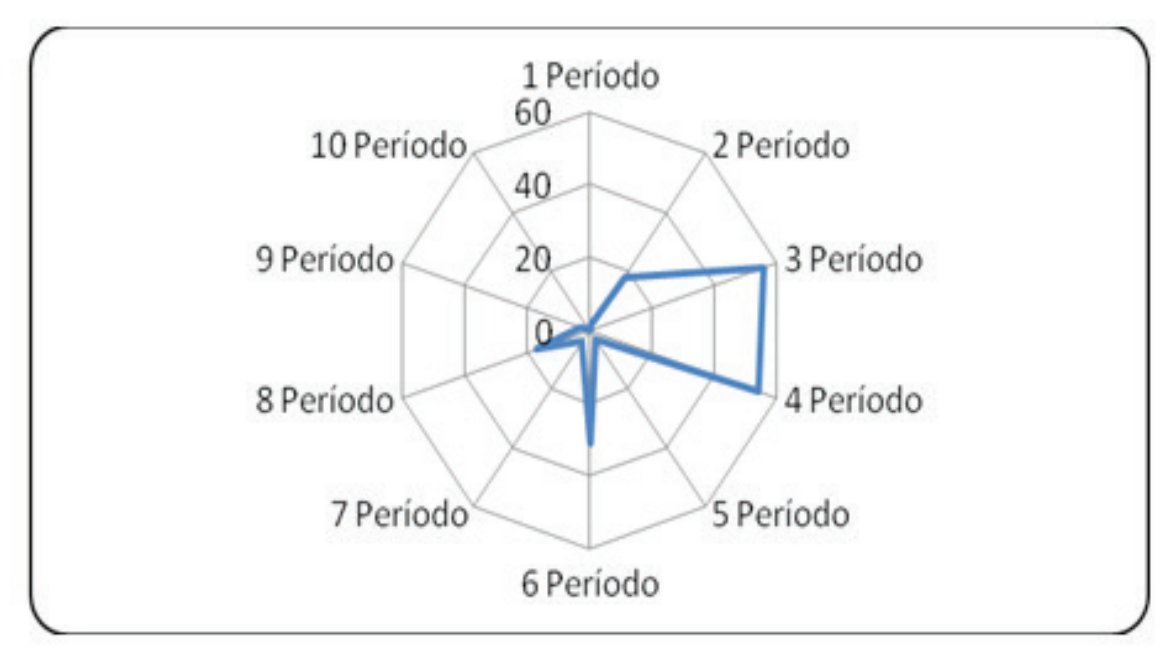

Figura 1. Distribuição dos estudantes da área de saúde, UFV (2013) nos seus respectivos períodos acadêmicos; 1 no primeiro período, 18 no segundo, 56 no terceiro, 54 no quarto, 3 no quinto, 31 no sexto, 4 no sétimo, 17 no oitavo e 3 no nono período.

Sobre a prática de atividade física, 16,58\% dos estudantes se autodeclararam sedentários, $49,73 \%$ pouco ativos, $28,74 \%$ moderadamente ativos e $5,35 \%$ se consideraram muito ativos. Estes dados estão de acordo com outros estudos que identificaram elevados índices de sedentarismo neste tipo de população ${ }^{24,26}$. Marcondelli, Costa, Schmitz 29 realizaram um estudo com 281 estudantes da área da saúde da Universidade de Brasília e encontraram alto índice de sedentarismo o qual foi justificado principalmente pela falta de tempo e de dinheiro para a realização dessas atividades. O alto percentual de estudantes sedentários ou pouco ativos não era esperado por se tratar de universitários da área de saúde e que, a princípio, possuem conhecimento sobre os benefícios da prática de atividade física na promoção da saúde.

De acordo com American College of Sports
Medicine uma atividade aeróbica moderada como a caminhada, por exemplo, de três a cinco vezes por semana, com duração de 30 minutos, já traz benefícios à saúde cardiovascular e, vale considerar, que tal prática não é onerosa e nem demorada.

Quanto à ingestão de nutrientes merece destaque a elevada ingestão média diária de açúcar de adição $(69,21 \pm 5,25$ g) que consequentemente, aumentou o teor de frutose da dieta $(36,22 \pm 29,78 \mathrm{~g})$ (Tabela 3$)$.

O percentual médio de açúcar de adição em relação ao VET da dieta foi de 15,37\%. Vale ressaltar que levando em consideração o valor de referência (10\%) foi observado que $75,35 \%$ dos estudantes estavam com a ingestão inadequada de açúcar de adição e que $13,37 \%$ dos voluntários apresentaram ingestão de frutose acima de $50 \mathrm{~g} / \mathrm{dia}$, desconsiderando a frutose não proveniente do açúcar de adição. 
Tabela 3. Ingestão média, desvio padrão, mediana com valor mínimo e máximo de macro e micronutrientes pelos estudantes da área de saúde, UFV, 2013.

\begin{tabular}{|c|c|c|c|c|c|c|}
\hline $\begin{array}{c}\text { Nutrientes } \\
(n=142)\end{array}$ & Média & $\begin{array}{l}\text { Desvio } \\
\text { Padrão }\end{array}$ & $\begin{array}{c}\text { Erro } \\
\text { Padrão }\end{array}$ & Mediana & $\begin{array}{c}\text { Valor } \\
\text { Mínimo }\end{array}$ & $\begin{array}{l}\text { Valor } \\
\text { Máximo }\end{array}$ \\
\hline Calorias (kcal) & 1757,0 & 583,87 & 48,99 & 1658,32 & 699,33 & 4274,12 \\
\hline Proteínas (g) & 66,80 & 22,528 & 1,89 & 64,36 & 25,52 & 165,73 \\
\hline Lipídios (g) & 55,71 & 26,06 & 2,18 & 50,09 & 16,88 & 187,54 \\
\hline Carboidratos (g) & 249,24 & 86,33 & 7,24 & 238,97 & 92,30 & 576,40 \\
\hline $\begin{array}{l}\text { Açúcar } \\
\text { de adição (g) }\end{array}$ & 69,21 & 46,3594 & 3,89 & 60,28 & 5,00 & 238,00 \\
\hline $\begin{array}{l}\text { CHO Total/ } \\
\text { Açúcar } \\
\text { de adição }\end{array}$ & 5,40 & 5,65 & 0,47 & 3,95 & 0,51 & 52,07 \\
\hline Frutose $(\mathrm{g})$ & 36,22 & 29,78 & 2,49 & 30,27 & 2,50 & 105,76 \\
\hline Cálcio (mg) & 548,75 & 298,68 & 25,06 & 510,11 & 89,66 & 1863,08 \\
\hline Vitamina C (mg) & 125,61 & 107,24 & 8,99 & 96,10 & 13,55 & 522,84 \\
\hline Sódio (mg) & 1771,42 & 824,01 & 69,14 & 1571,40 & 421,82 & 4963,37 \\
\hline Fibras Totais (g) & 15,51 & 7,30 & 0,63 & 14,51 & 2,39 & 48,10 \\
\hline Fibra insolúvel (g) & 4,51 & 2,91 & 0,24 & 3,96 & 0,69 & 18,36 \\
\hline Fibra solúvel (g) & 2,66 & 1,76 & 0,14 & 2,22 & 0,26 & 10,15 \\
\hline
\end{tabular}

CHO: carboidratos.

Tem sido observado que a ingestão de alimentos de alta carga glicêmica pode contribuir para o desenvolvimento de inflamação sistêmica subclínica, resistência insulínica e prejuízo na função das células beta pancreáticas, podendo resultar em alteração da tolerância à glicose e DM2. Além disso, o elevado teor de frutose dessas bebidas pode aumentar o risco de desenvolvimento de doenças cardiovasculares devido à dislipidemia, aumento da gordura visceral, hipertensão arterial e hiperuricemia ${ }^{4,7}$.

Outro desfecho metabólico importante tem sido o observado sobre os níveis plasmáticos de lipídios em adultos e crianças com ingestão elevada de açúcar de adição e frutose na dieta habitual ${ }^{30}$. Em um estudo com adultos americanos com ingestão de açúcar de adição acima de $10 \%$ do valor calórico total (VCT) da dieta foram observados níveis plasmáticos baixos de HDL colesterol e elevados níveis de triglicerídeos e LDL colesterol, representando um perfil lipídico de maior risco para o desenvolvimento de doença coronariana ${ }^{31}$.

Sabe-se que a ingestão de dieta rica em frutose está associada à maior taxa da lipogênese de novo e maiores níveis plasmáticos de lipídios em humanos. O fígado é o principal sítio da lipogênese de novo, e é responsável pela utilização da maior parte da frutose da dieta que alcança a veia porta. Entretanto, o mecanismo molecular subjacente ao aumento da produção hepática de lipídios em resposta a dieta de alto teor de frutose permanece desconhecido ${ }^{30,32}$.

Quanto aos micronutrientes analisados, a ingestão de cálcio representou 68,6\% da recomendação ${ }^{33}$ para esta faixa etária. A baixa adequação do consumo de cálcio proveniente da alimentação poderá favorecer o desenvolvimento de patologias, incluindo câncer, doenças infecciosas, inflamatórias e autoimunes, cardiovasculares e metabólicas, como obesidade e hipertensão ${ }^{34}$.

Foi verificada também uma ingestão média diária de 15,51 \pm 7,30g de fibras totais na dieta, que corresponde a $62,35 \%$ da recomendação da OMS de 25 gramas/dia. Para os outros nutrientes avaliados, não houve inadequações no consumo médio dos estudantes.
101

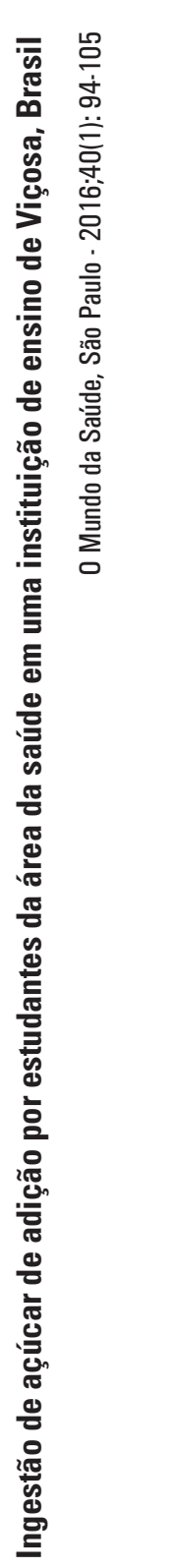


Outros estudos têm mostrado que os cursos de graduação da área de saúde têm influenciado pouco nos hábitos de vida desta população ${ }^{26,35}$.

Uma importante consequência do consumo de uma dieta habitual com elevado teor de açúcar simples é a redução da ingestão de micronutrientes, podendo resultar em inadequações dietéticas quando tal hábito alimentar é adotado desde a infância ${ }^{1}$. Neste sentido, avaliando-se a ingestão de refrigerantes e sucos de frutas entre adolescentes na Suécia, os resultados de um estudo mostraram que aqueles indivíduos com ingestão elevada de refrigerantes e sucos de frutas, apresentavam também ingestão reduzida de leite e derivados, resultando em dieta pobre em cálcio e prejuízo da densidade mineral óssea ${ }^{36}$.

No que tange ao estudo das correlações, verificou-se que a ingestão média de açúcar de adição esteve significativamente $(p<0,05)$ associada ao peso corporal $(0,172 ; p=0,041)$, IMC $(0,181 ; p=0,031)$, além do consumo dos macronutrientes e do cálcio $(0,247 ; p=0,003)$ (Tabela 4).

Um estudo transversal com adultos canadenses saudáveis avaliou a ingestão habitual de carboidratos dos participantes através do registro alimentar de 24 horas aplicado durante 3 dias não consecutivos. Observou-se que os voluntários que ingeriram habitualmente dieta com menor teor de açúcar simples apresentaram menor tendência ao sobrepeso e obesidade. Entretanto, os participantes cuja ingestão de carboidratos totais da dieta correspondia a menos que $47 \%$ do VET ingerido apresentaram maior risco de ganho ponderal do que aqueles cujo teor de carboidratos da dieta habitual apresentava-se entre 47 a $64 \%$ do VCT ingerido ${ }^{37}$. Tal resultado poderia ser explicado pela menor ingestão de fibras, frutas e vegetais, e maior ingestão de lipídios observada entre os indivíduos com ingestão habitual de carboidratos totais abaixo de $47 \%$ do VCT.

Tabela 4. Coeficientes de correlação de Pearson dos fatores associados à ingestão média de açúcar de adição consumido por estudantes da área de saúde, UFV, 2013.

\begin{tabular}{lcc}
\hline \multicolumn{1}{c}{ Variáveis avaliadas (n= 142) } & Coeficiente de Correlação & $\mathbf{p}$ \\
Período acadêmico & $-0,059$ & 0,488 \\
\hline Idade (anos) & 0,118 & 0,165 \\
Peso $(\mathrm{kg})$ & 0,172 & $0,041^{*}$ \\
\hline IMC (kg.m-2) & 0,181 & $0,031^{*}$ \\
CC (cm) & 0,151 & 0,076 \\
\hline Calorias (kcal) & 0,613 & $0,000^{*}$ \\
Proteínas (g) & 0,282 & $0,001^{*}$ \\
\hline Lipídios (g) & 0,349 & $0,000^{*}$ \\
Carboidratos (g) & 0,700 & $0,000^{*}$ \\
\hline CHO Total & $-0,487$ & $0,000^{*}$ \\
Açúcar de adição & 0,762 & $0,000^{*}$ \\
Frutose (g) & & \\
\hline Cálcio (mg) & 0,247 & $0,003^{*}$ \\
Vitamina C (mg) & $-0,008$ & 0,924 \\
\hline Sódio (mg) & 0,049 & 0,560 \\
Fibras Totais (g) & 0,026 & 0,768 \\
\hline Fibra insolúvel (g) & 0,087 & 0,301 \\
Fibra solúvel (g) & $-0,020$ & 0,816 \\
\hline
\end{tabular}

CHO: carboidratos; ${ }^{*} \mathrm{p}<0,05$ 
Resultados semelhantes foram observados na Austrália em um estudo que realizou a revisão de dados epidemiológicos obtidos a partir de entidades de nutrição e saúde do governo australiano. Foi observado que, apesar da ingestão alimentar de carboidratos apresentar-se em declínio nos últimos anos, o desenvolvimento de sobrepeso e obesidade continuou aumentando ${ }^{38}$. A possível explicação para tal paradoxo pode residir no fato de que a redução no consumo de carboidratos totais desta população tenha sido acompanhada de substituição da ingestão destes nutrientes na dieta habitual por açúcar refinado ou carboidratos de alto índice glicêmico, gordura saturada ou álcool.

É importante ressaltar que, cada vez mais, resultados de grande número de estudos tem demonstrado a relevância da qualidade e não só da quantidade de carboidratos da dieta ingerida ${ }^{32}$. Verifica-se em grande parte dos estudos que a ingestão de grãos integrais, legumes e frutas que são fontes mais adequadas de carboidratos da dieta está associada à redução do risco de desenvolvimento de doenças cardiovasculares. Por outro lado, alimentos ricos em açúcar de adição e xarope de milho rico em frutose, especialmente sob a forma de refrigerantes, têm sido cada vez mais associados ao desenvolvimento de doenças crônicas não transmissíveis ${ }^{39}$.

Corroborando com estes dados, foi observado que a redução de $355 \mathrm{ml} /$ dia no consumo de refrigerantes e outras bebidas ricas em açúcar de adição por adultos saudáveis, correspondeu a uma redução de 1,8/1,1 mmHg na relação da pressão arterial sistólica/diastólica nestes indivíduos, independentemente do teor de cafeína presente neste tipo de bebida ${ }^{40}$. De maneira semelhante, em um estudo com adolescentes saudáveis, a avaliação da dieta habitual por questionários de frequência alimentar revelou níveis plasmáticos mais elevados de ácido úrico e maiores níveis da pressão arterial sistólica entre os adolescentes que consumiam quantidades mais elevadas de bebidas ricas em sacarose e frutose ${ }^{41}$.

Atualmente, tem sido verificada tendência significativa na redução dos teores de açúcar simples da dieta ocidental ${ }^{31,38}$. Um estudo avaliando dados epidemiológicos do governo americano observou redução da ingestão de açúcar de adição da dieta, principalmente, às custas do menor consumo de refrigerantes ${ }^{31}$. Embora tenha sido verificada tal redução na ingestão de açúcar de adição na dieta dos participantes, a ingestão média deste nutriente ainda continuou excedendo os limites recomendados.

Em outro estudo longitudinal com 1689 adultos americanos, foi orientada a substituição de refrigerantes da dieta habitual por bebidas de baixa caloria no período de 1999 a $2004^{42}$. Após análise retrospectiva dos dados do estudo em questão, foi verificada perda ponderal significativa e melhora da qualidade da dieta dos participantes ao longo do período do estudo, mostrando que abordagens dietéticas simples também são capazes de trazer benefícios à saúde da população.

Acredita-se que todos os esforços para a redução da ingestão habitual de açúcar de adição e excesso de frutose na dieta ocidental possam resultar em melhora dos parâmetros metabólicos de adultos e crianças, alcançandose, deste modo, a prevenção das doenças crônicas não transmissíveis ${ }^{31,42}$. O presente estudo possui relevância uma vez que o comportamento alimentar de estudantes da área de saúde estabelecidos durante o período da juventude pode ter impacto significante na ocorrência de doenças futuras.

\section{CONCLUSÃO}

Os dados deste estudo revelaram o desequilíbrio na alimentação de universitários dos cursos de saúde. Foi verificada uma elevada ingestão de açucares de adição além de uma baixa ingestão de cálcio e fibras alimentares. Também foi verificada a associação deste padrão alimentar com sobrepeso e outros aspectos importantes que são fatores de risco para algumas doenças crônicas não transmissíveis. Portanto, é de se considerar a criação de comissões que avaliem a capacidade dos cursos universitários de saúde a promoverem mudanças comportamentais, importantes no que diz respeito à alimentação e programas de educação em saúde.
103 


\section{REFERÊNCIAS}

1. Steyn NP et al. Evidence to support a food-based dietary guideline on sugar consumption in South Africa. BMC Public Health. 2012; 12:502.

2. Sonestedt $\mathrm{E}$ et al. Does high sugar consumption exacerbate cardiometabolic risk factors and increase the risk of type 2 diabetes and cardiovascular disease? Food \& Nutrition Research. 2012;56:19104.

3. Chun OC, Chung CE, Wang Y, Padgitt A, Song WO. Changes in Intakes of Total and Added Sugar and their Contribution to Energy Intake in the U.S. Nutrients. 2010; 2:834-54.

4. Hu FB, Malik VS. Sugar-sweetened beverages and risk of obesity and type 2 diabetes: Epidemiologic evidence. Physiol Behav. 2010;

5. U.S. Department of Health and Human Services; U.S. Department of Agriculture. Dietary Guidelines for Americans. Washington DC: Government Printing Office; 2005.

6. World Health Organization. WHO. Diet, nutrition and the prevention of chronic diseases. Geneva: World Health Organization, 2003.

7. Stanhope KL, Havel PJ. Endocrine and metabolic effects of consuming beverages sweetened with fructose, glucose, sucrose, or high fructose corn syrup. Am J Clin Nutr. 2008; 88(6):1733S-7S.

8. Lustig RH. Fructose: Metabolic, Hedonic, and Societal Parallels with Ethanol. J Am Diet Assoc. 2010;110:1307-21.

9. Brasil. Ministério da Saúde. Secretaria de Atenção à Saúde. Guia alimentar para a população brasileira: promovendo a alimentação saudável. Brasília: Ministério da Saúde; 2006.

10. Instituto Brasileiro de Geografia e Estatística (2010) Pesquisa de Orçamentos Familiares 2008-2009: Avaliação Nutricional da Disponibilidade Domiciliar de Alimentos no Brasil. Rio de Janeiro: IBGE.

11. Cluskey M, Grobe D. College weight gain and behavior transitions: male and female differences. J Am Diet Assoc [Intenet]. 2009 [acesso 2012 out 07];109:325-329. Disponível em: http://www.sciencedirect.com/science/article/pii/S0002822308020385 Doi: 10.1016/j.jada.2008.10.045.

12. Verger P, Combes JB, Kovess-Masfety V, Choquet M, Guagliardo V, Rouillon F, et al. Psychological distress in first year university students: socioeconomic and academic stressors, mastery and social support in young men and women. Social Psych Psychiatric Epidemiology, 2009; 44:643-650. doi: 10.1007/s00127-008-0486-y.

13. American College Health Association. American College Health Association-National College Health Assessment II: Reference Group Executive Summary, Spring 2010. Linthicum, MD: American College Health Association [Internet]. 2010 [acesso 2012 ago 23]. Disponível em : <http://www.acha-ncha.org/docs/ACHA-NCHA-II_ReferenceGroup_ExecutiveSummary_Fall2010.pdf>.

14. Souza LB, Malta MB, Donato PM, Corrente JE, Carvalhaes MABL, Papini SJ. Inadequação de consumo alimentar, antropometria e estilo de vida de universitárias da área de saúde. J Health Sci Inst 2012; 30(4):377-81. DOI:10.1016/S0140-6736 (11)60135-9. 10.

15. Souza DP. Avaliação do estado nutricional e consumo alimentar de acadêmicos do curso de nutrição da Universidade Federal de Pelotas, Rev HCPA 2012; 32(3):275-282.

16. Monteiro MRP, Andrade MLO, Zanirati VF, Silva RR. Hábito e consumo alimentar de estudantes do sexo feminino dos cursos de Nutrição e de Enfermagem de uma universidade pública brasileira. Rev APS 2009; 12(3):271-277.

17. Universidade Federal de Viçosa (UFV). Disponível em: [http://www.cpd.ufv.br/relatorioufv] Acessado em: 19 de outubro de 2012. 18. Institute of Medicine; Food and Nutrition Board. Dietary reference intakes for energy, carbohydrate, fiber, fat, fatty acids, cholesterol, protein and amino acids. Washington: National Academy Press, 2002.

19. Zabotto CB, Veanna RPT, Gil MF. Registro fotográfico para inquéritos dietéticos: utensilios e porções. Goiânia : Nepa-Unicamp; 1996.

20. Johnson AA et al. Dietary intakes, anthropometric measurements and pregnancy outcomes. J Nutr.; 94:936S-42S.

21. Stanhope KL. et al. Consumption of fructose and high fructose corn syrup increase postprandial triglycerides, LDL-cholesterol and apolipoprotein-B in young men and women. J Clin Endoclin Metab. 2011; 96:10.

22. WHO (World Health Organization). Obesity: Preventing and Managing the Global Epidemic. Report of a WHO Consultation on Obesity. Geneva: WHO. 1998.

23. WHO (World Health Organization). Physical status: the use and interpretation of anthopometry. In: Technical Report Series; 1995. World Health Organization. Disponível em http://www.who.int/tecnicalreportseries. Acesso em 04 de setembro de 2015.

24. Costa KCBC et al. Insatisfação corporal em estudantes universitários da área de saúde nos Estados de Alagoas e Sergipe. Mudanças - Psicologia da Saúde. 2010; 18(1-2):1-6.

25. Lucas ACS et al. Uso de psicotrópicos entre universitários da área da saúde da Universidade Federal do Amazonas, Brasil. Cad. Saúde Pública. 2006; 22(3):663-71.

26. Franca C, Colares V. Estudo comparativo de condutas de saúde entre universitários no início e no final do curso. Rev. Saúde Pública. 2008; 42.

27. Paixão LA da, Dias RMR, Prado WL do. Estilo de vida e estado nutricional de universitários ingressantes em cursos da área de saúde do recife/PE. Rev Bras Ativ Fís Saúde. 2010;15(3):145-50.

28. Oliveira, RAR de. Variáveis bioquímicas, antropométricas e pressóricas como indicadores de risco cardiovascular em servidores públicos. Fisioter Mov. 2013;26(2):369-77.

29. Marcondelli P, Costa T, Schimtz IB. Nível de atividade física e hábitos alimentares de universitários do 3 ao 5 semestres da área de saúde. Rev Nutr. 2008; 21(1):39-47.

30. Hirahatake KM, Meissen JK, Fiehn O, Adams SH. Comparative Effects of Fructose and Glucose on Lipogenic Gene Expression and Intermediary Metabolism in HepG2 Liver Cells. Plos One. 6(11).

31. Welsh JA et al. Caloric Sweetener Consumption and Dyslipidemia Among US Adults. JAMA. 2010; 21; 303(15):1490-7.

32. Chong MFF, Fielding BA, Frayn KN. Mechanisms for the acute effect of fructose on postprandial lipemia. Am J Clin Nutr. 2007; 85:1511-20.

33. Institute of Medicine; Food and Nutrition Board. Dietary reference intakes for calcium and vitamin D. Washington: National Academy Press, 2012.

34. Leão ALM, Santos LC dos. Consumo de micronutrientes e excesso de peso: existe relação? Rev Bras Epidemiol. 2012;15(1):85-95. 35. Steptoe A et al. Trends in smoking, diet, physical exercise, and attitudes toward health in European university students from 13 
countries, 1990-2000. Prev Med. 2002; 35(2):97-104.

36. Vagstrand K et al. Correlates of soft drink and fruit juice consumption among Swedish adolescents. Br J Nutr. 2009; 101(10):154148.

37. Merchant AT et al. Carbohydrate Intake And Overweight And Obesity Among Healthy Adults. J Am Diet Assoc. 2009;109 (7):1165-72.

38. Barclay AW, Brand-Miller J. The Australian Paradox: A Substantial Decline in Sugars Intake over the Same Timeframe that Overweight and Obesity Have Increased. Nutrients. 2011;3:491-504.

39. Aller EEJ, Abete I, Astrup A, Martinez JA, Van Baak MA. Starches, Sugars and Obesity. Nutrients. 2012, 3:341-69.

40. Brown I J. et al. Sugar-Sweetened Beverage, Sugar Intake Of Individuals And Their Blood Pressure: Intermap Study. Hypertension. 2011; 57(4):695-701.

41. Nguyen S, Choi HK, Lusting RH, Hsu C. Sugar Sweetened Beverages, Serum Uric Acid, and Blood Pressure in Adolescents. J Pediatr. 2009; 154(6):807-13.

42. Thomson JL et al. Simulated reductions in consumption of sugar-sweetened beverages improves diet quality in Lower Mississippi Delta adults. Food \& Nutrition Research. 2011;55:7304. 